\title{
Sarilumab and adalimumab differential effects on bone remodelling and cardiovascular risk biomarkers, and predictions of treatment outcomes
}

Cem Gabay ${ }^{1}$, Gerd R. Burmester ${ }^{2}$, Vibeke Strand ${ }^{3}$, Jérôme Msihid ${ }^{4}$, Moshe Zilberstein ${ }^{5}$, Toshio Kimura ${ }^{6}$, Hubert van Hoogstraten ${ }^{5}$, Susan H. Boklage ${ }^{6}$, Jonathan Sadeh ${ }^{5}$, Neil M. H. Graham ${ }^{6}$ and Anita Boyapati ${ }^{6 *}$

\begin{abstract}
Background: Interleukin-6 (IL-6) is a pleiotropic cytokine that plays a key role in the pathogenesis of rheumatoid arthritis. Sarilumab is a human monoclonal antibody that binds membrane-bound and soluble IL-6 receptor-a to inhibit IL-6 signalling. The aim of this study was to compare the effects of sarilumab and adalimumab (a tumour necrosis factor alpha inhibitor) monotherapy on levels of circulating biomarkers associated with the acute-phase response, bone remodelling, atherothrombosis, anaemia of chronic disease and markers purported to reflect synovial lymphoid and myeloid cell infiltrates, as well as the potential of these biomarkers to differentially predict clinical and patient-reported outcomes with sarilumab vs. adalimumab.
\end{abstract}

Methods: In this post hoc analysis, serum samples were analysed at baseline and prespecified post-treatment timepoints up to week 24 in adults with moderate-to-severe active rheumatoid arthritis intolerant of or inadequate responders to methotrexate from the MONARCH trial (NCT02332590).

Results: Greater reductions in C-reactive protein (CRP; $-94.0 \%$ vs. $-24.0 \%$ ), serum amyloid A (SAA; $-83.2 \%$ vs. $-17.4 \%$ ), total receptor activator of nuclear factor-KB ligand (RANKL; $-18.3 \%$ vs. 10.5\%) and lipoprotein (a) (- $41.0 \%$ vs. $-2.8 \%)$ were observed at week 24 with sarilumab vs. adalimumab, respectively (adjusted $p<0.0001$ ). Greater increases in procollagen type $1 \mathrm{~N}$-terminal propeptide (P1NP) were observed with sarilumab vs. adalimumab at week 24 (22.8\% vs. $6.2 \%, p=0.027$ ). Patients with high baseline SAA, CRP and matrix metalloproteinase-3 (MMP-3) were more likely to achieve clinical efficacy, including American College of Rheumatology 20\% improvement criteria and Disease Activity Score (28 joints)-CRP < 3.2, and report improvements in patient-reported outcomes, including Health Assessment Questionnaire-Disability Index and pain visual analogue scale, with sarilumab than adalimumab.

Conclusion: Sarilumab was associated with greater positive effects on bone remodelling and decreases in biomarkers of the acute-phase response, synovial inflammation and cardiovascular risk vs. adalimumab. High baseline concentrations of SAA, CRP and MMP-3 are predictive of clinical and patient-reported outcome responses to sarilumab treatment and prospective validation is warranted to confirm these results.

(Continued on next page)

\footnotetext{
* Correspondence: anita.boyapati@regeneron.com

${ }^{6}$ Regeneron Pharmaceuticals Inc., Tarrytown, NY, USA

Full list of author information is available at the end of the article
}

(C) The Author(s). 2020 Open Access This article is licensed under a Creative Commons Attribution 4.0 International License, which permits use, sharing, adaptation, distribution and reproduction in any medium or format, as long as you give appropriate credit to the original author(s) and the source, provide a link to the Creative Commons licence, and indicate if changes were made. The images or other third party material in this article are included in the article's Creative Commons licence, unless indicated otherwise in a credit line to the material. If material is not included in the article's Creative Commons licence and your intended use is not permitted by statutory regulation or exceeds the permitted use, you will need to obtain permission directly from the copyright holder. To view a copy of this licence, visit http://creativecommons.org/licenses/by/4.0/ The Creative Commons Public Domain Dedication waiver (http://creativecommons.org/publicdomain/zero/1.0/) applies to the data made available in this article, unless otherwise stated in a credit line to the data. 
(Continued from previous page)

Trial registration: ClinicalTrials.gov, NCT02332590. Registered on 5 January 2015

Keywords: Rheumatoid arthritis, Sarilumab, Biomarkers, Biologic disease-modifying antirheumatic drug, Cardiovascular risk, Bone remodelling, Acute-phase response, Synovial inflammation

\section{Background}

Patients with rheumatoid arthritis (RA) develop bone and cartilage damage in synovial joints as a result of chronic inflammation, which is mediated by proinflammatory cytokines such as interleukin-6 (IL-6) and tumour necrosis factor alpha (TNF- $\alpha$ ) [1-3]. In RA, elevated circulating cytokine concentrations trigger bone and cartilage destruction through activation of signalling cascades that lead to the stimulation of osteoclasts via bone-resorptive factors (e.g. receptor activator of nuclear factor- $\mathrm{kB}$ ligand [RANKL]) and joint-destructive proteins (e.g. matrix metalloproteinases) [3, 4]. Underlying joint damage leads to long-term impairments in physical function [5].

IL-6 is a pleiotropic cytokine that plays a role in inflammatory, metabolic, neural and regenerative processes [6]. IL-6 operates through two distinct mechanisms-classic (cis) and trans-signalling-which expands the range of its actions and contributes towards the systemic manifestations and co-morbidities commonly associated with RA, including the acute-phase response, osteoporosis, fatigue, depression, anaemia and cardiovascular (CV) disease [7-9]. Patients with RA have an increased risk of CV events, including myocardial infarction and stroke, relative to healthy individuals [10]. It is understood that pro-inflammatory cytokines promote endothelial dysfunction and structural vessel abnormalities and induce other CV risk factors, including changes in lipid levels, insulin resistance and oxidative stress [11]. In addition, significantly elevated levels of lipoprotein (a) (Lp [a]), a biomarker of $\mathrm{CV}$ risk which is involved in both inflammation and thrombosis, have been observed in patients with RA compared with healthy controls [12].

Sarilumab is a human monoclonal antibody that binds membrane-bound and soluble IL- 6 receptor- $\alpha$ to inhibit IL- 6 signalling. It is approved for the treatment of adults with moderate-to-severe active RA as monotherapy and in combination with conventional synthetic disease-modifying antirheumatic drugs (csDMARDs) [13-15]. Adalimumab is a human monoclonal antibody that blocks TNF- $\alpha$ and is approved for the treatment of RA, among other conditions [16]. The efficacy of sarilumab vs. adalimumab was evaluated in the MONARCH phase III randomized controlled trial (NCT02332590) [15]. Sarilumab monotherapy was superior to adalimumab monotherapy, as demonstrated by greater reduction in Disease Activity Score (28 joints) using erythrocyte sedimentation rate (DAS28-ESR) in adults with moderate-to-severe active RA who were intolerant of or inadequate responders to methotrexate (MTX-INT/MTX-IR) $[15,17]$. The safety profiles of both therapies were consistent with anticipated class effects.

In this post hoc analysis, levels of circulating biomarkers were evaluated at baseline and after treatment, which were associated with: (1) the acute-phase response (C-reactive protein [CRP] and serum amyloid A [SAA]), (2) bone remodelling (procollagen type $1 \mathrm{~N}$-terminal propeptide [P1NP], osteocalcin [OC], total RANKL and osteoprotegerin [OPG]), (3) synovial inflammation (matrix metalloproteinase-3 [MMP-3]), (4) purported to reflect synovial lymphoid (chemokine [C-X-C motif] ligand 13 [CXCL13]) and myeloid cell (soluble intercellular adhesion molecule-1 [sICAM-1]) infiltrates, (5) atherothrombosis (Lp [a]) and (6) anaemia of chronic disease (hepcidin, ferritin, total iron-binding capacity [TIBC] and iron). The effects of sarilumab and adalimumab monotherapy on the levels of these markers were examined, as well as the potential of these markers at baseline to differentially predict the efficacy of or improvement in patientreported outcomes (PROs) with sarilumab vs. adalimumab. This analysis did not evaluate the relationship between biomarkers and safety parameters.

\section{Methods}

This phase III active-comparator randomized controlled trial has been described in full previously [15]. In brief, MTX-INT/IR patients were randomized to sarilumab $200 \mathrm{mg}$ every 2 weeks (q2w) or adalimumab $40 \mathrm{mg} \mathrm{q} 2 \mathrm{w}$ for 24 weeks. At week 16, dose escalation to weekly adalimumab was permitted for those who did not achieve $\geq 20 \%$ improvement in tender and swollen joint counts. The trial was conducted in accordance with Good Clinical Practice and with the principles of the Declaration of Helsinki; all protocols and patient information materials were approved by appropriate ethical review boards and all patients provided written informed consent.

\section{Efficacy and PRO endpoints}

Efficacy endpoints included the following: proportion of patients achieving $\geq 20 / 50 / 70 \%$ improvement according to American College of Rheumatology criteria (ACR20/ 50/70), Clinical Disease Activity Index (CDAI) $\leq 2.8$, CDAI $\leq 10$, DAS28 using CRP (DAS28-CRP) or DAS28ESR $<2.6$ and DAS28-CRP or DAS28-ESR $<3.2$.

PROs evaluated in the study were previously described for the overall intent-to-treat (ITT) population [17] and, 
evaluated as change from baseline at week 24, included Patient Global Assessment of disease activity visual analogue scale (VAS), Health Assessment QuestionnaireDisability Index (HAQ-DI), pain VAS, Functional Assessment of Chronic Illness Therapy (FACIT)-Fatigue, morning stiffness VAS, rheumatoid arthritis impact of disease (RAID) score and Medical Outcomes Study ShortForm (36-item) Health Survey (SF-36) physical (PCS) and mental (MCS) component summary scores, which include the physical functioning, role-physical, bodily pain, general health, vitality, social functioning, role-emotional and mental health domains.

\section{Serum collection and biomarker analysis}

Patients were selected for this biomarker analysis if they had been randomized and treated with sarilumab or adalimumab during the double-blind period and had provided written informed consent for future use of samples, with a serum sample drawn pre-dose (baseline) and evaluable (biomarker population). Serum samples were collected and stored frozen at baseline and post-treatment through week 24 from 307 patients in the ITT population (sarilumab, $n=153$; adalimumab, $n=154)$.

Biomarkers were analysed retrospectively (except CRP) at one or two post-baseline timepoints through week 24 (Table S1). Timepoints selected for analysis were based on either previous data following sarilumab treatment $[18,19]$ or on literature suggesting either acute or latent effects of RA therapy on specific markers. The assay characteristics for most biomarkers have been described previously [18].

\section{Statistical analysis}

Baseline biomarker levels were compared between treatment groups using a Wilcoxon test. Spearman's ranked correlations at baseline were computed in the overall biomarker population.

To evaluate pharmacodynamic changes in circulating biomarker concentrations between treatment groups at each timepoint, absolute and percentage changes from baseline were described. In addition, the percentage changes in biomarker concentrations were analysed using non-parametric methods because of non-normal distributions. For biomarkers measured once postbaseline, a rank-based analysis of covariance (ANCOVA) adjusted on baseline value was implemented. For biomarkers measured twice post-baseline, a mixed-effect model with repeated measures was performed on ranktransformed data (analysis of variance [ANOVA]-type method), with treatment, visit and treatment-by-visit interaction as fixed effects, baseline biomarker value transformed in rank, and baseline biomarker value transformed in rank-by-visit interaction as fixed covariates, assuming an unstructured covariance structure. The logtransformed RANKL/OPG ratio was analysed using a mixed model for repeated measures with response, visit and response-by-visit interaction as fixed effects, baseline biomarker value and baseline biomarker value-byvisit interaction as fixed covariates, and assuming an unstructured covariance structure separately by treatment group. $p$ values were adjusted for false discovery rate (Benjamini-Hochberg 5\% threshold). The number of patients with abnormal biomarker levels at baseline (according to the reference ranges provided by the testing laboratory) that normalized with treatment was compared between groups using a $\chi^{2}$ test; nominal $p$ values are reported.

Subgroup analyses were performed according to the use of systemic steroids at baseline. Percentage changes from baseline in biomarker levels were analysed separately in each subgroup, and nominal $p$ values were provided.

Percentage changes in biomarker concentrations at week 24 were compared between clinical responders and non-responders at the same visit within each treatment group using similar non-parametric methods. $p$ values were also adjusted for false discovery rate.

For binary efficacy endpoints, predictive effects of baseline biomarker values on sarilumab efficacy vs. adalimumab were tested using a logistic regression with treatment group and region as fixed effects, baseline biomarker value as a continuous covariate and the baseline biomarker-by-treatment group interaction. For continuous PROs, a linear regression was used with the same effects as above, as well as the baseline PRO value as a covariate. Nominal values for the interaction are reported to assess the predictive value of the biomarkers. Similar analyses were performed after categorization of patients into high, medium and low biomarker levels at baseline using tertile values in the biomarker population. In addition, pairwise comparisons of responses between sarilumab and adalimumab were performed separately in patients with high, medium and low biomarker levels, and the Mantel-Haenszel estimates of odds ratios (ORs), stratified by region, and $95 \%$ confidence intervals (CIs) were derived and graphically represented using forest plots. For continuous PROs, a linear regression was performed separately in each biomarker tertile and differences in least squares mean (LSM) changes with 95\% CI between both treatments were provided.

Differential combinations of circulating CXCL13 and sICAM-1 (low or high levels defined relative to baseline median levels) were assessed for prediction of response to sarilumab, using Mantel-Haenszel estimates of ORs derived for each combination.

All analyses were performed using SAS version 9.2 or higher (SAS Institute Inc., Cary, NC, USA). 


\section{Results}

Baseline demographics, disease characteristics, efficacy and biomarker levels

Baseline demographics and disease characteristics of the biomarker population were generally similar to the overall ITT population (Table 1). Overall, efficacy and PROs were also generally similar between the ITT and biomarker populations (Table S2).

Baseline serum levels of most biomarkers were similar between treatment groups, except for Lp(a), which was higher in the adalimumab than the sarilumab groups (Lp [a]: median 235.5 vs. $179.0 \mathrm{mg} / \mathrm{L}$, respectively; Wilcoxon test $p$ value 0.039 ; Table S3).

Correlations between individual biomarkers at baseline were generally low or moderate $(\rho<0.5$; Fig. 1$)$. Correlation coefficients above 0.7 were observed for markers of inflammation (CRP and SAA; $\rho=0.81$ ), bone formation (P1NP and OC; $\rho=0.82$ ) and anaemia of chronic disease (ferritin and hepcidin; $\rho=0.74$ ), as expected. Moderate correlations were observed between baseline CRP, SAA or MMP-3 with differential blood counts (leucocytes and neutrophils; $\rho$ from 0.4 to 0.5 ) and, as expected, between iron and haemoglobin $(\rho=0.57$; Figure S1).

\section{Pharmacodynamic effects of treatment on biomarkers}

To compare the effects of sarilumab and adalimumab treatment on biomarkers over time, the absolute (Table S4) and percentage changes from baseline in biomarker concentrations were analysed up to week 24. Greater reductions in biomarkers associated with the acute-phase response were observed at weeks 12 and 24 following treatment with sarilumab vs. adalimumab (adjusted $p<0.0001$; Fig. 2a, b). Reductions in CRP were observed as early as week 4 with sarilumab vs. adalimumab and were sustained throughout the treatment period (Fig. 2a for median percentage

Table 1 Baseline patient demographics and disease characteristics

\begin{tabular}{|c|c|c|c|c|}
\hline & ITT population & & Biomarker population & \\
\hline & $\begin{array}{l}\text { Adalimumab } 40 \mathrm{mg} \\
\mathrm{q} 2 \mathrm{w}(n=185)\end{array}$ & $\begin{array}{l}\text { Sarilumab } 200 \mathrm{mg} \\
\mathrm{q} 2 \mathrm{w}(n=184)\end{array}$ & $\begin{array}{l}\text { Adalimumab } 40 \mathrm{mg} \\
\mathrm{q} 2 \mathrm{w}(n=154)\end{array}$ & $\begin{array}{l}\text { Sarilumab } 200 \mathrm{mg} \\
\mathrm{q} 2 \mathrm{w}(n=153)\end{array}$ \\
\hline Age, years & $53.6(11.9)$ & $50.9(12.6)$ & $53.3(12.0)$ & $50.4(12.5)$ \\
\hline Sex, female, \% & 81.1 & 85.3 & 78.6 & 83.7 \\
\hline Duration of RA, years & $6.6(7.8)$ & $8.1(8.1)$ & $6.6(8.1)$ & $7.9(8.1)$ \\
\hline ACPA positive, $\%$ & 76.7 & 75.3 & 76.2 & 73.8 \\
\hline RF positive, \% & 64.8 & 66.9 & 63.8 & 66.0 \\
\hline $\begin{array}{l}\text { Treated with } 1 / 2 / \geq 3 \text { prior csDMARDs/ } \\
\text { immunosuppressive agents, } \%\end{array}$ & $47.6 / 31.4 / 21.1$ & $45.1 / 31.0 / 23.9$ & $48.1 / 31.8 / 20.1$ & $46.4 / 32.0 / 21.6$ \\
\hline Oral corticosteroid use, \% & 56.2 & 53.3 & 57.1 & 51.6 \\
\hline Tender joint count & $26.7(13.6)$ & $28.0(13.2)$ & $26.9(13.9)$ & $28.1(13.4)$ \\
\hline Swollen joint count & $17.5(10.3)$ & $18.6(10.7)$ & $17.3(10.1)$ & $18.5(10.6)$ \\
\hline HAQ-DI & $1.6(0.6)$ & $1.6(0.6)$ & $1.6(0.6)$ & $1.6(0.6)$ \\
\hline CRP, mg/L & $24.1(31.0)$ & $17.4(21.3)$ & $23.6(31.1)$ & $17.4(21.9)$ \\
\hline DAS28-ESR & $6.8(0.8)$ & $6.8(0.8)$ & $6.8(0.8)$ & $6.8(0.8)$ \\
\hline DAS28-CRP & $6.0(0.9)$ & $6.0(0.9)$ & $6.0(0.9)$ & $6.0(0.9)$ \\
\hline CDAl & $42.4(12.0)$ & $43.6(12.1)$ & $42.8(11.9)$ & $43.8(12.0)$ \\
\hline Pain VAS (0-100 mm) & $71.4(19.0)$ & $71.6(18.7)$ & $71.3(18.6)$ & $71.2(19.1)$ \\
\hline Patient global VAS (0-100 mm) & $67.8(18.4)$ & $68.0(17.5)$ & $68.6(18.2)$ & $67.8(17.9)$ \\
\hline SF-36 PCS & $31.4(6.6)$ & $30.7(6.2)$ & $31.5(6.5)$ & $30.4(6.2)$ \\
\hline SF-36 MCS & $37.1(11.8)$ & $36.7(10.7)$ & $37.4(12.4)$ & $37.0(11.2)$ \\
\hline Morning stiffness VAS (0-100 mm) & $68.0(21.4)$ & $70.8(19.0)$ & $68.2(21.7)$ & $70.6(18.8)$ \\
\hline FACIT-fatigue (0-52) & $24.0(10.3)$ & $24.0(9.0)$ & $23.9(10.4)$ & $23.4(9.1)$ \\
\hline Physician global VAS (0-100 mm) & $66.0(17.1)$ & $66.3(15.7)$ & $66.2(16.4)$ & $67.3(14.9)$ \\
\hline RAID (0-10) & $6.4(2.0)$ & $6.7(1.7)$ & $6.4(2.1)$ & $6.6(1.7)$ \\
\hline
\end{tabular}

Mean (standard deviation) unless otherwise stated

ACPA anti-citrullinated protein antibody, CDAl Clinical Disease Activity Index, CRP C-reactive protein, csDMARD conventional synthetic disease-modifying antirheumatic drug, DAS28-CRP Disease Activity Score (28 joints) using C-reactive protein, DAS28-ESR Disease Activity Score (28 joints) using erythrocyte sedimentation rate, FACIT Functional Assessment of Chronic Illness Therapy, HAQ-DI Health Assessment Questionnaire-Disability Index, ITT intent-to-treat, MCS mental component summary, PCS physical component summary, $q 2 w$ every 2 weeks, $R A$ rheumatoid arthritis, $R A I D$ rheumatoid arthritis impact of disease, $R F$ rheumatoid factor, SF-36 Medical Outcomes Study Short-Form (36-item) Health Survey, VAS visual analogue scale 


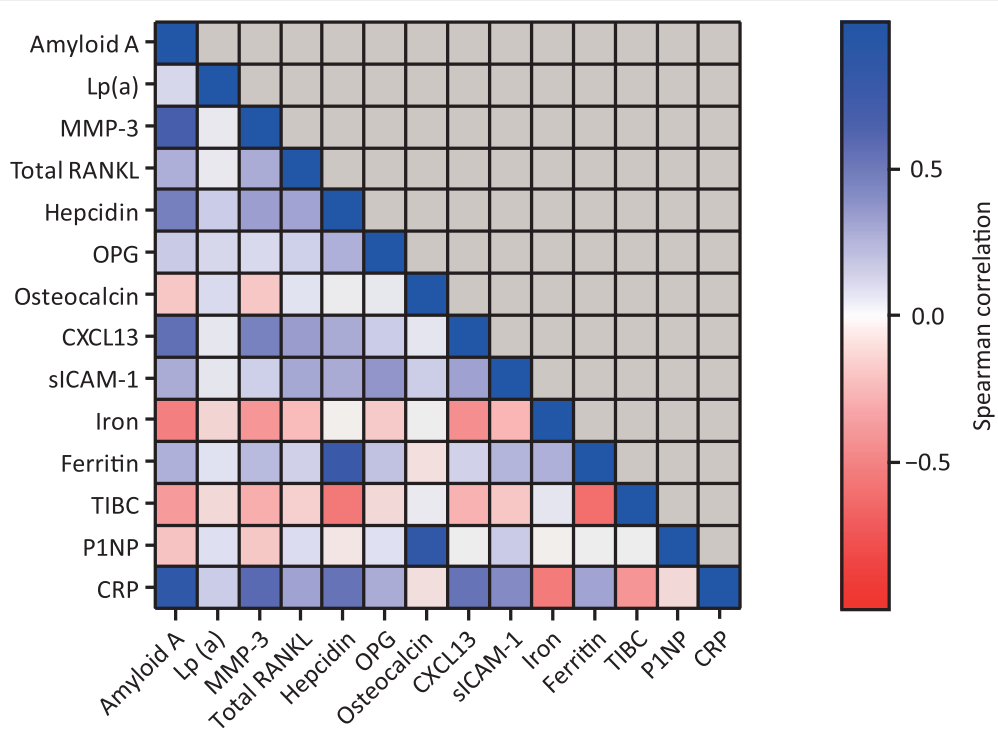

Fig. 1 Correlations between baseline biomarkers. CRP, C-reactive protein; CXCL13, chemokine (C-X-C motif) ligand 13; Lp(a), lipoprotein (a); MMP3, matrix metalloproteinase-3; OPG, osteoprotegerin; P1NP, procollagen type $1 \mathrm{~N}$-terminal propeptide; RANKL, receptor activator of nuclear factorKB ligand; SAA, serum amyloid A; SICAM-1, soluble intercellular adhesion molecule-1; TIBC, total iron-binding capacity

change in CRP from baseline in biomarker population from week 4; Table S5 for percentage of patients with CRP $\leq 10 \mathrm{mg} / \mathrm{L}$ and $\leq 3 \mathrm{mg} / \mathrm{L}$ at weeks 12 and 24 [observed cases within the ITT population]).

At week 24, sarilumab treatment increased concentrations of P1NP, a marker of osteoblast activation, compared with adalimumab (adjusted $p=0.027$; Fig. 2c). A numeric increase in $\mathrm{OC}$, another marker of osteoblast activity, was also observed in sarilumab- vs. adalimumabtreated patients (Fig. 2d). Furthermore, reductions in total RANKL, a marker of bone remodelling, were observed as early as week 2 with sarilumab compared with adalimumab and persisted through week 24 (adjusted $p<0.0001$; Fig. 2e); in addition, a numeric increase in total RANKL was observed after adalimumab treatment. A transient decrease in OPG, a decoy for RANKL, was observed after adalimumab treatment at week 2 but did not persist through week 24 (Fig. 2f). The log of the ratio of RANKL to OPG was also significantly decreased in patients treated with sarilumab vs. adalimumab (data not shown). Subgroup analyses revealed that this effect was significant through week 24 in patients who were not on steroids at baseline (nominal $p=0.0013$ ). Additionally, greater reductions in MMP-3 were observed with sarilumab at week 24 (adjusted $p=0.020$; Fig. 2g). Since corticosteroid use impacts bone remodelling, we compared the treatment effects in subgroups based on baseline corticosteroid use. At week 24 the differential effects of sarilumab on MMP-3 concentrations were significant (nominal $p=0.001$ ) only in the subgroup of patients (sarilumab: $n=79,51.6 \%$; adalimumab: $n=88,57.1 \%$ ) who were not on concomitant steroids (data not shown). Reductions in total RANKL were significant in the sarilumab treatment group compared with adalimumab treatment irrespective of steroid use at baseline (nominal $p<0.01$ ). Increases in P1NP, though numerically increased by sarilumab treatment relative to adalimumab, did not reach statistical significance in the steroid subgroups (median percentage change from baseline in P1NP [interquartile range]: with baseline steroid use, $24.6 \%$ [ -0.6 to $50.3 \%$ ] with sarilumab and $7.5 \%$ [ -9.9 to $40.3 \%$ ] with adalimumab [nominal $p=0.0757$ ]; without baseline steroid use, $18.0 \%$ [ -3.5 to $47.2 \%$ ] with sarilumab and $1.8 \%$ [- 13.9 to $31.9 \%$ ] with adalimumab [nominal $p=0.1625]$ ).

The effects of sarilumab and adalimumab on biomarkers associated with markers purported to reflect synovial lymphoid and myeloid cell infiltrates, CXCL13 and sICAM-1, respectively, were also examined. While greater reductions in these biomarkers were observed 2 weeks post-treatment with adalimumab vs. sarilumab, these effects did not persist through week 24 (Figure S2).

We also examined the effects of treatment on parameters associated with anaemia of chronic disease. Previously unpublished data showed that, in the overall safety population (sarilumab, $n=184$; adalimumab, $n=185$ ), sarilumab resulted in larger increases in haemoglobin levels vs. adalimumab [20] from baseline (mean 13.0 and $12.9 \mathrm{~g} / \mathrm{dL}$, respectively) at week 12 (LSM changes from baseline 0.53 vs. $0.12 \mathrm{~g} / \mathrm{dL}$, respectively; LSM difference $0.41 \mathrm{~g} / \mathrm{dL}$ [95\% CI 0.22-0.60; nominal $p<0.001]$ ) and week 24 (LSM changes from baseline 0.59 vs. $0.08 \mathrm{~g} / \mathrm{dL}$, respectively; LSM difference $0.52 \mathrm{~g} / \mathrm{dL} \quad[95 \%$ CI $0.32-0.71$; nominal $p<0.001]$ ). Furthermore, in the overall ITT population, a numerically greater reduction from 

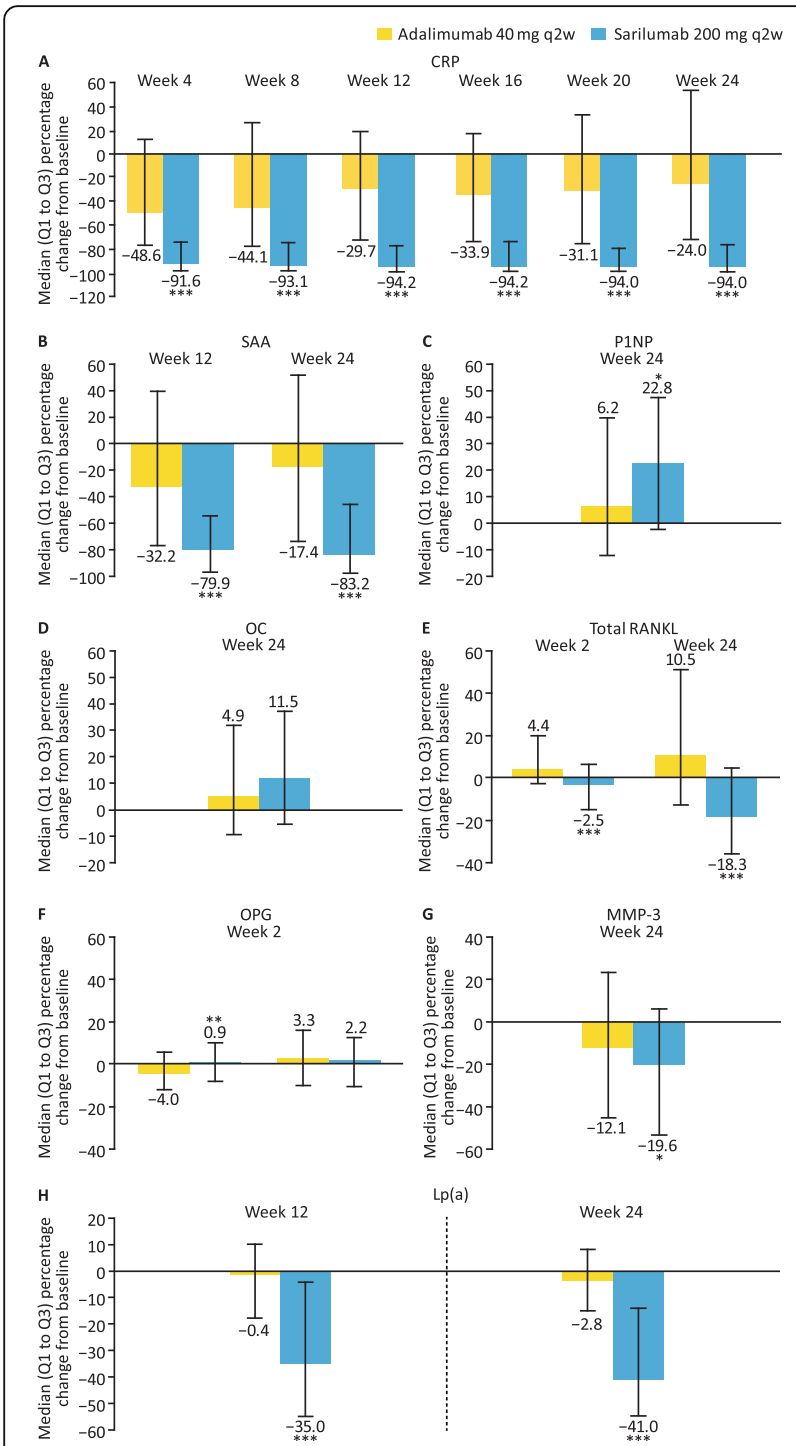

Lp(a)

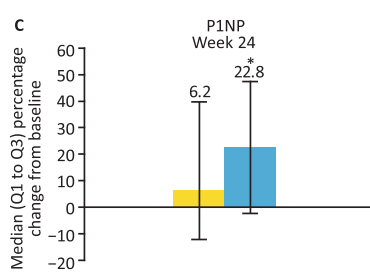

Week 16 Week 20 Week 24

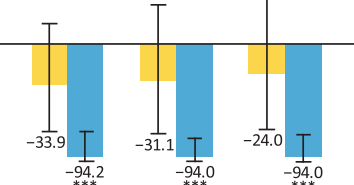

Adalimumab $40 \mathrm{mg}$ q2w Sarilumab $200 \mathrm{mg}$ q2w

Lp(a) were observed with sarilumab vs. adalimumab at week 24 (adjusted $p<0.0001$; Fig. 2h).

A subset of patients had abnormal baseline biomarker levels relative to reference ranges. In these patients, normalization of CRP and SAA was evident in a greater percentage treated with sarilumab than adalimumab at week 24 (nominal $p<0.0001$ ). Normalization of total RANKL, OPG and $\mathrm{Lp}(\mathrm{a})$ occurred in a numerically greater percentage of patients treated with sarilumab vs. adalimumab at week 24 (Fig. 3).

\section{Relationship between changes in biomarker levels and clinical responses}

To establish whether post-treatment changes in biomarker levels at week 24 were associated with clinical efficacy, changes were compared between sarilumab- and adalimumab-treated responders and non-responders. Median percentage changes at week 24 in total RANKL, OPG, P1NP, OC and Lp(a) did not differ greatly between responders and non-responders (data not shown). However, reductions in SAA from baseline at week 24 were greater in adalimumab ACR20 and DAS28-CRP $<3.2$ responders than non-responders $(-33.3 \%$ vs. $0.0 \%$, respectively; nominal $p=0.0038$ and $-39.2 \%$ vs. $0.0 \%$, respectively; nominal $p=0.0061$, respectively). Greater reductions in MMP-3 were also observed in adalimumab ACR20 responders vs. non-responders $(-23.6 \%$ vs. $17.1 \%$, respectively; nominal $p<0.0001)$. Associations between clinical efficacy and changes from baseline in SAA and MMP-3 were not observed in sarilumab-treated patients, and although both responders and non-responders had a $\geq 90 \%$ reduction in CRP, the $p$ values for comparisons of responders vs. non-responders were $>0.05$ across several parameters, including ACR20/50, DAS28-CRP $<3.2$ and DAS28-CRP $<2.6$ (data not shown).

Fig. 2 Median percentage changes from baseline in biomarkers through week 24. Median percentage changes from baseline in biomarkers of $\mathbf{a}, \mathbf{b}$ the acute-phase response (CRP and SAA), $\mathbf{c}-\mathbf{f}$ bone remodelling (P1NP, OC, total RANKL and OPG), $\mathbf{g}$ synovial inflammation (MMP-3) and $\mathbf{h}$ atherothrombosis (Lp [a]). *Adjusted $p<0.05$; **adjusted $p<0.01$ vs. adalimumab; ${ }^{* * *}$ adjusted $p<0.0001$ vs. adalimumab (Benjamini-Hochberg procedure). CRP, C-reactive protein; Lp(a), lipoprotein (a); MMP-3, matrix metalloproteinase-3; OC, osteocalcin; OPG, osteoprotegerin; P1NP, procollagen type $1 \mathrm{~N}$-terminal propeptide; $\mathrm{Q}$, quartile; $\mathrm{q} 2 \mathrm{~W}$, every 2 weeks; RANKL, receptor activator of nuclear factor-KB ligand; SAA, serum amyloid A

baseline in the proportion of patients with anaemia was observed with sarilumab vs. adalimumab from baseline: reductions were observed as early as week 2 and persisted through week 24 (Table S6). In this post hoc analysis, reductions in hepcidin and ferritin were observed at week 2 with both sarilumab and adalimumab. In contrast, increases in iron and TIBC were observed with sarilumab relative to adalimumab at week 2 posttreatment (Figure S3). Reductions in the lipid particle

\section{Correlations between biomarkers and disease activity and PROs at baseline}

The strongest correlations between baseline biomarkers and baseline disease activity were observed for SAA and CRP with DAS28-ESR ( $\rho=0.26$ and 0.31 , respectively) and for CRP, SAA, MMP-3, hepcidin and CXCL13 with DAS28-CRP ( $\rho$ from 0.36 to 0.58 ). None of the biomarkers correlated with baseline PROs (all $\rho<0.3$ ).

\section{Predictive analysis of baseline biomarker levels on clinical responses and PROs}

Baseline biomarker levels were analysed as continuous and categorical measures by tertiles (low, medium and high) because thresholds associated with clinical efficacy are not currently established, and treatment-bybiomarker interaction $p$ values were calculated to assess the predictivity of the biomarker. Treatment-by-tertile biomarker interactions for efficacy endpoints at week 24 


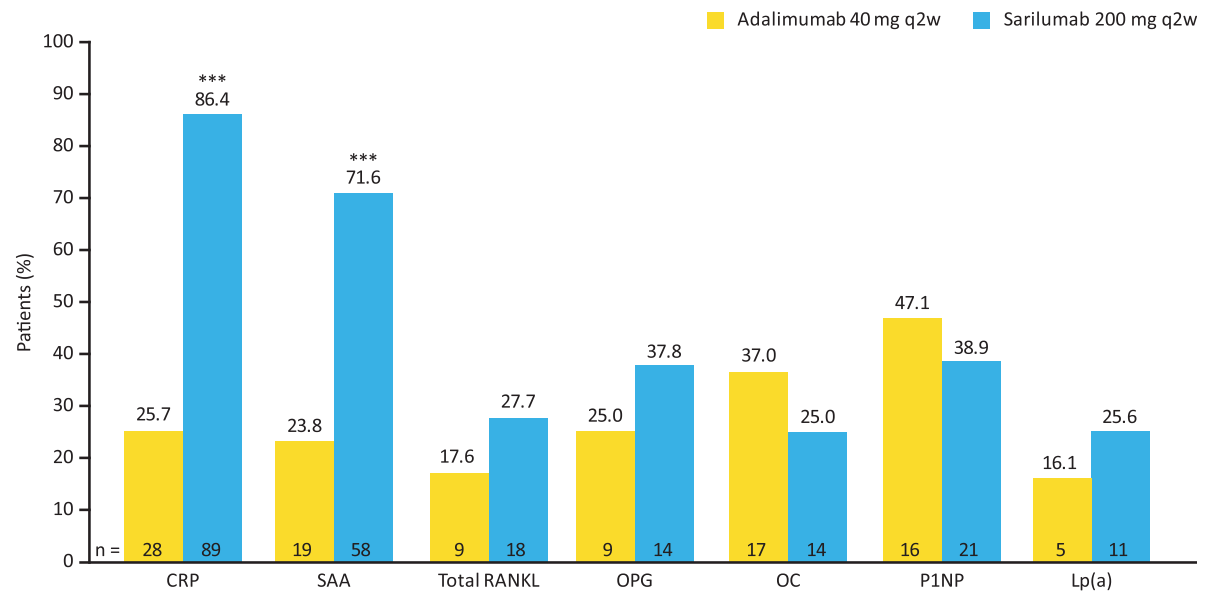

Fig. 3 Proportions of patients whose biomarker values returned to normal reference ranges at week 24. Proportions of patients with biomarker serum concentrations exceeding the reference range at baseline that normalized to within reference range at week 24 . ${ }^{* * *}$ Nominal $p<0.0001$ vs. adalimumab ( $X^{2}$ test). CRP, C-reactive protein; Lp(a), lipoprotein (a); OC, osteocalcin; OPG, osteoprotegerin; P1NP, procollagen type $1 \mathrm{~N}$-terminal propeptide; q2W, every 2 weeks; RANKL, receptor activator of nuclear factor-kB ligand; SAA, serum amyloid A

analysed by baseline biomarker in tertiles are shown in Fig. 4 and Table S7. Patients with the highest baseline concentrations of SAA who received sarilumab were more likely to achieve ACR20/50/70 or DAS28-CRP $<3.2$ responses than with adalimumab compared with patients in the low tertile: ACR20 (OR [95\% CI] 5.5 [2.1, 14.5]), ACR50 (5.4 [2.2, 13.2]), ACR70 (5.7 [1.8, 18.4]) and DAS28-CRP < $3.2(6.1[2.3,15.7])$ (Fig. 4 and Table S7). SAA was consistently predictive compared with high MMP-3 and CRP, which were only predictive of ACR20 and DAS28-CRP $<3.2$ response (Table S7). Baseline levels of biomarkers associated with bone remodelling, synovial lymphoid and myeloid cell infiltrates and anaemia of inflammation were not predictive of efficacy at week 24, except for hepcidin and CXCL13, which were associated with ACR20 response.

The ability of baseline biomarker levels to predict PRO responses was also analysed by their respective tertiles and showed that sarilumab-treated patients with higher SAA, MMP-3 and hepcidin levels reported improved

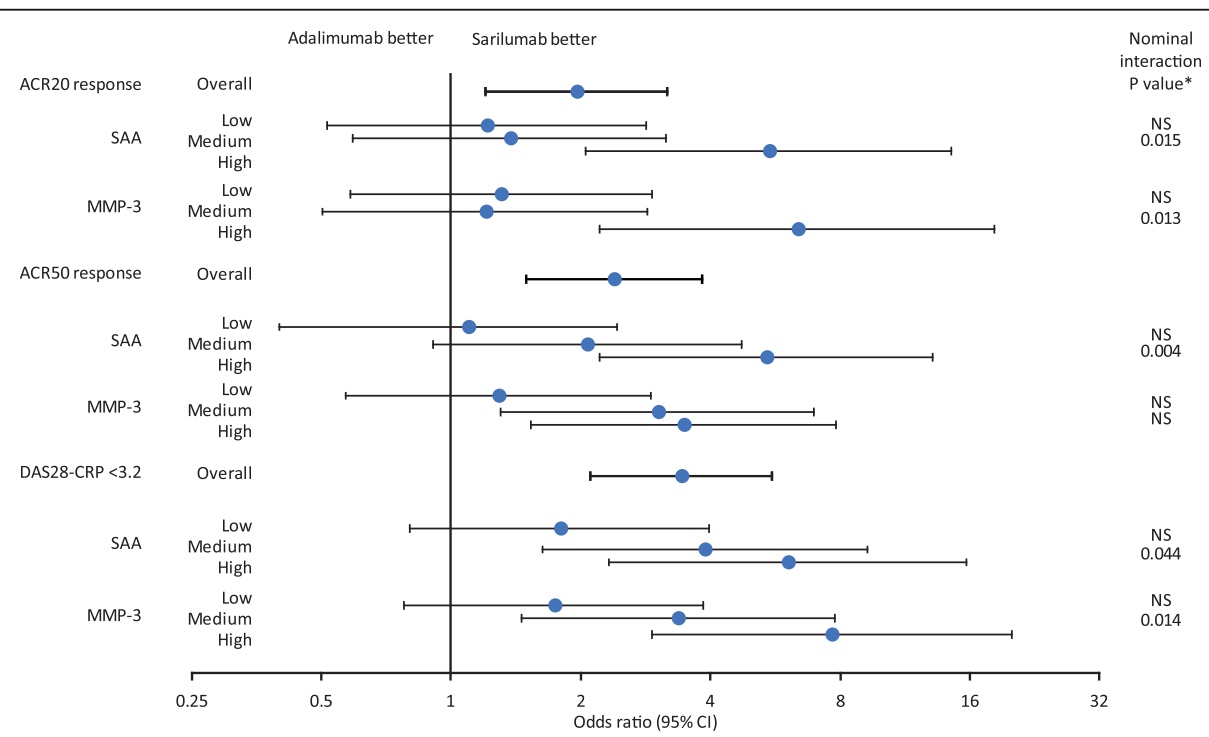

Fig. 4 Odds ratios for achieving efficacy endpoints at week 24 by baseline biomarker tertile. Odds ratios (sarilumab vs. adalimumab) for achieving ACR20, ACR50 and DAS28-CRP $<3.2$ responses at week 24 by baseline biomarker tertile. *Nominal biomarker-by-treatment interaction vs. low tertile. Low, medium and high subgroups are based on biomarker tertile values in overall treatment groups (see Table S3 for tertile ranges). ACR20/50, American College of Rheumatology 20/50\% improvement criteria; Cl, confidence interval; DAS28-CRP, Disease Activity Score (28 joints) using C-reactive protein; MMP-3, matrix metalloproteinase-3; NS, not significant at 5\%; SAA, serum amyloid A 
PRO responses including HAQ-DI (Fig. 5a) and pain VAS (Fig. 5b) scores compared with adalimumab-treated patients, as well as patient global VAS, morning stiffness VAS, SF-36 PCS and physical functioning domain and RAID. The $p$ values for these interactions are included in Fig. 5 and Table S8 to demonstrate the differential efficacy predicted by high levels of these biomarkers compared with low levels. Baseline levels of markers associated with anaemia of chronic disease (hepcidin, ferritin and iron) were also associated with PRO improvements at week 24 (Table S8). Analysis of biomarkers as continuous measures also revealed interactions for SAA, MMP-3, CRP and P1NP with HAQ-DI at week 24 (interaction nominal $p$ values $<0.01$ ).

\section{Evaluation of differential combinations of markers associated with myeloid and lymphoid activation for prediction of clinical response}

Baseline levels of CXCL13 and sICAM-1 were analysed to determine whether differential ratios of these biomarkers (high/high, high/low, low/high and low/low; using the median in the overall population as the cutoff) could predict clinical responses to sarilumab or adalimumab treatment at week 24. While CXCL13 high/ sICAM-1 high and CXCL13 low/sICAM-1 low patients had greater ACR50 responses with sarilumab than adalimumab, the other combinations were not predictive (nominal $p>0.05$; Figure S4). Additionally, CXCL13 high/sICAM-1 high patients had greater ACR20 responses with sarilumab than adalimumab (OR 3.8 [95\% CI 1.5, 9.8]; nominal $p=0.004)$ but other combinations were not predictive (nominal $p>0.05$ ).

\section{Discussion}

Analysis of the pharmacodynamic effects of treatment on circulating biomarkers found sarilumab treatment reduced biomarkers of the acute-phase response, bone remodelling, synovial inflammation and CV risk and increased bone formation markers compared with adalimumab. These effects were generally observed early and persisted through to week 24 . This was particularly evident with CRP and is consistent with previous observations $[13,14,18,19]$. In addition, a greater proportion of patients treated with sarilumab vs. adalimumab monotherapy demonstrated normalization of serum biomarkers at week 24, which was greatest for biomarkers of the acute-phase response (CRP and SAA).

In this study, a limited number of bone remodelling markers were evaluated. Inhibitors of bone formation induced by the Wnt pathway such as DKK-1 and sclerostin are upregulated in patients with RA and have been shown to be decreased by anti-IL- 6 and anti-TNF therapies [21]. The pharmacodynamic effects of sarilumab

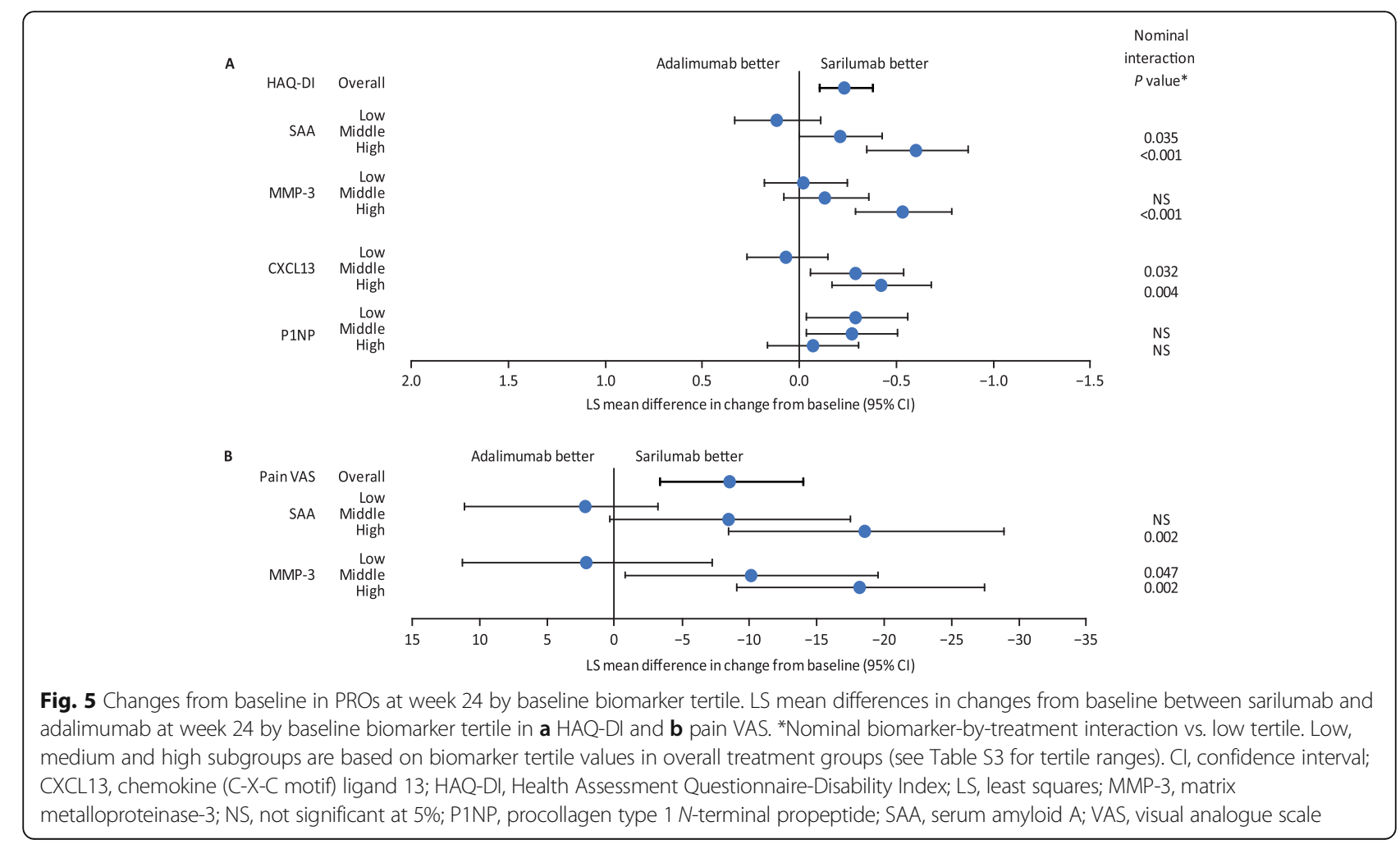


monotherapy observed in MONARCH are generally consistent with those reported after 24 weeks treatment in the MOBILITY (MTX-IR patients treated with sarilumab $150 \mathrm{mg} / 200 \mathrm{mg}$ q2w + MTX vs. placebo + MTX) and TARGET (TNF-IR patients treated with sarilumab $150 \mathrm{mg} / 200 \mathrm{mg} \mathrm{q} 2 \mathrm{w}+$ csDMARDs vs. placebo + csDMARDs) biomarker populations, where sarilumab treatment reduced biomarkers of bone resorption and synovial inflammation $[18,19]$. P1NP was significantly increased after sarilumab monotherapy compared with adalimumab, but in other studies, TNF inhibition has been associated with significant increases in P1NP posttreatment [22].

Limited data in patients with RA suggest that the IL-6 receptor blocker tocilizumab was more effective than TNF- $\alpha$ inhibitors in improving anaemia of chronic disease and normalizing iron metabolism via inhibition of hepcidin [23]. As hepcidin is involved in regulating iron stores in macrophages, a reduction in hepcidin is associated with greater iron availability. We observed increases in both iron and TIBC levels with sarilumab vs. adalimumab treatment despite similar effects of both on hepcidin and ferritin.

In our analysis, given that both SAA and CRP are regulated by IL-6, biomarkers of the acute-phase response were strongly correlated with DAS28-CRP at baseline (nominal $p<0.0001$ ). However, no correlations were observed between baseline biomarkers and PROs. Reductions in several biomarkers were associated with clinical efficacy at week 24 in adalimumabtreated patients; however, these associations were not observed in the sarilumab group. This result suggests that IL-6 receptor blockade may have a direct effect on production of these biomarkers independent of its effects on disease activity, in contrast to TNF inhibitors.

Biomarkers that can predict responses to treatment would be valuable in supporting the paradigm shift towards personalized medicine [24-26]. In this study, univariate analysis identified several biomarkers that individually predicted responses to sarilumab treatment. Upon further analysis, high levels of CRP, SAA, MMP-3, CXCL13 and hepcidin predicted ACR20 responses to sarilumab. Several of these markers are included in the multi-biomarker disease activity (MBDA) score which is associated with disease activity but has not been evaluated for differential response to specific biologic therapies [27, 28]. Baseline levels of markers associated with anaemia of chronic disease did not predict efficacy (except hepcidin); however, these markers were associated with changes in several PROs. High baseline levels of SAA and MMP-3 were also associated with improvements in several PROs, including patient global VAS, HAQ-DI, pain VAS, SF-36 PCS and MCS scores, morning stiffness VAS and RAID score.

Dennis et al. observed that patients with greater ACR50 response rates following treatment with the IL-6 inhibitor tocilizumab had higher baseline lymphoid relative to myeloid activity [27]. Conversely, patients with higher myeloid synovial signatures responded better to adalimumab, suggesting that baseline characteristics may predict therapeutic responses to agents with different mechanisms of action. We also evaluated whether differential levels of CXCL13 and sICAM-1 predicted ACR50 responses in MONARCH. We used similar methodology to Dennis et al. (defining low and high levels using the pre-treatment median as the cut-off) but owing to different biomarker levels in patient populations, medians were not comparable, and our results did not replicate the previous findings. This may also be attributed to baseline differences in synovial phenotypes between the two trial populations and/or differences in the assays used to measure these markers. Synovial biopsies were not performed before initiating therapy in either trial, and although synovial phenotypes can predict differential responses, other markers reflective of lymphoid and myeloid cells may require measurement to capture underlying synovial phenotypes and pathology.

Interpretations of this analysis should acknowledge these data are up to week 24 following treatment initiation and the limited sampling of biomarkers (many only at baseline and weeks 2 and 24); further exploration of more frequent and/or later timepoints may provide a more complete picture of biomarker changes following therapy. It is also important to recognize that these analyses were restricted to circulating biomarkers accounting for only a portion of total biomarker matter; additional approaches are needed to understand the effects of treatment on synovial biomarkers. Finally, this was a post hoc analysis of patients well defined according to inclusion and exclusion criteria of the trial; therefore, additional analyses are required to further understand the effect of sarilumab treatment on these biomarkers in a wider population of RA patients.

\section{Conclusion}

In this analysis, sarilumab treatment was associated with decreases in circulating biomarkers of the acute-phase response, bone resorption, synovial inflammation and CV risk compared with adalimumab. Several biomarkers, including MMP-3, SAA and CRP, were associated with clinical efficacy and individually predicted response to sarilumab treatment. Further studies evaluating the predictive value of changes in these biomarkers are necessary to confirm these findings and identify patients more likely to respond to sarilumab administration. 


\section{Supplementary information}

Supplementary information accompanies this paper at https://doi.org/10. 1186/s13075-020-02163-6.

Additional file 1. Additional methodology. Table S1. Individual serum biomarker assessment schedule. Table S2. Efficacy and PROs at week 24 in the biomarker and ITT populations. Table S3. Baseline biomarker serum concentrations in the biomarker population. Table S4. Absolute change from baseline in biomarker concentrations through week 24. Table S5. Percentage of patients with CRP $\leq 10 \mathrm{mg} / \mathrm{L}$ and $\leq 3 \mathrm{mg} / \mathrm{L}$ at weeks 12 and 24 (overall safety population). Table S6. Percentage of patients with anaemia at weeks 2 and 24 (overall safety population). Table S7. Treatment-by-tertile biomarker interactions for efficacy endpoints at week 24 analysed by baseline biomarker in tertiles. Table S8. Treatment-by-tertile biomarker interactions for PROs at week 24 analysed by baseline biomarker in tertiles. Figure S1. Correlation matrix for baseline biomarkers and haematology parameters. Figure S2. Median percentage changes from baseline in (A) CXCL13 and (B) sICAM-1 through week 24 . Figure S3. Median percentage changes from baseline in biomarkers of anaemia of chronic disease 2 weeks post treatment. Figure S4. ACR50 responses at week 24 and corresponding ORs with differential combinations of CXCL13 and sICAM-1.

\section{Abbreviations}

ACPA: Anti-citrullinated protein antibody; ACR20/50/70: American College of Rheumatology 20/50/70\% improvement criteria; ANCOVA: Analysis of covariance; ANOVA: Analysis of variance; CDAI: Clinical Disease Activity Index; $\mathrm{Cl}$ : Confidence interval; CRP: C-reactive protein; CSDMARD: Conventional synthetic disease-modifying anti-rheumatic drug; CXCL13: Chemokine (C-X-C motif) ligand 13; DAS28-CRP: Disease Activity Score (28 joints) using Creactive protein; CV: Cardiovascular; DAS28-ESR: Disease Activity Score (28 joints) using erythrocyte sedimentation rate; FACIT: Functional Assessment of Chronic IIIness Therapy; HAQ-DI: Health Assessment Questionnaire-Disability Index; IL-6: Interleukin-6; ITT: Intent-to-treat; Lp(a): Lipoprotein (a); LS: Least squares; LSM: Least squares mean; MBDA: Multi-biomarker disease activity; MCS: Mental component summary; MMP-3: Matrix metalloproteinase-3; MTXINT/MTX-IR: Intolerant of or inadequate responders to methotrexate; OC: Osteocalcin; OPG: Osteoprotegerin; OR: Odds ratio; P1NP: Procollagen type $1 \mathrm{~N}$-terminal propeptide; PCS: Physical component summary; PRO: Patient-reported outcome; q2w: Every 2 weeks; RA: Rheumatoid arthritis; RAID: Rheumatoid arthritis impact of disease; RANKL: Receptor activator of nuclear factor-kB ligand; RF: Rheumatoid factor; SAA: Serum amyloid A; SF36: Medical Outcomes Study Short-Form (36-item) Health Survey; sICAM1: Soluble intercellular adhesion molecule-1; SJC: Swollen joint count; TIBC: Total iron-binding capacity; TJC: Tender joint count; TNF-a: Tumour necrosis factor alpha; VAS: Visual analogue scale

\section{Acknowledgements}

The authors thank the patients and their families, as well as the investigators and other study staff involved in the study.

\section{Authors' contributions}

$C G$ and $A B$ are responsible for the design and development of these analyses. GB is responsible for the acquisition of the data. All authors analysed or interpreted the data, critically revised the manuscript and are accountable for accuracy and integrity. The authors read and approved the final manuscript.

\section{Authors' information}

Dr. Sadeh was working at Sanofi Genzyme, Bridgewater, NJ, USA, for the study duration, data analyses, and the majority of the manuscript development, and has since left the company. Dr. Sadeh is currently employed by Bristol Myers Squibb.

\section{Funding}

Sanofi Genzyme and Regeneron Pharmaceuticals. Inc. funded the study, the analyses and medical writing support by Natalie Roberts, PhD, Adelphi Communications Ltd.

\section{Availability of data and materials}

Qualified researchers may request access to patient-level data and related study documents, including the clinical study report, study protocol with any amendments, blank case report form, statistical analysis plan and dataset specifications. Patient-level data will be anonymized and study documents will be redacted to protect the privacy of trial participants. Further details on Sanofi's data-sharing criteria, eligible studies and process for requesting access can be found at https://www.clinicalstudydatarequest.com.

\section{Ethics approval and consent to participate}

Protocols for all trials were approved by the appropriate ethics committees/ institutional review boards, and each patient provided written informed consent before participation in their study. The studies were conducted in compliance with institutional review board regulations, the International Conference on Harmonisation Guidelines for Good Clinical Practice and the Declaration of Helsinki.

\section{Consent for publication}

Not applicable

\section{Competing interests}

$C G$ received research grants from $A B 2$ Bio, Pfizer and Roche and consulting fees or other remuneration from AB2 Bio, AbbVie, Bristol Myers Squibb, Merck, Novartis, Pfizer, Regeneron, Roche, Sanofi and UCB. GRB has received research grants from AbbVie, Pfizer, UCB and Roche and consulting fees or other remuneration from AbbVie, Lilly, MSD, Pfizer, Sanofi, Roche and UCB. VS received consulting fees from AbbVie, Amgen, AstraZeneca, Biogen, Bristol Myers Squibb, Celltrion, CORRONA, Crescendo, Genentech/Roche, GlaxoSmithKline, Janssen, Eli Lilly, Novartis, Pfizer, Regeneron Pharmaceuticals Inc., Sandoz, Sanofi and UCB. JM is an employee of Sanofi and may hold stock and/or stock options in the company. $\mathrm{MZ}$ and $\mathrm{HvH}$ are employees of Sanofi Genzyme and may hold stock and/or stock options in the company. JS is a former employee of Sanofi Genzyme and may hold stock and/or stock options in the company and is currently employed by Bristol Myers Squibb. TK, SHB, NMHG and AB are employees of Regeneron Pharmaceuticals, Inc. and may hold stock and/or stock options in the company.

\section{Author details}

${ }^{1}$ University Hospitals of Geneva, Geneva, Switzerland. ${ }^{2}$ Charité - University Medicine Berlin, Berlin, Germany. ${ }^{3}$ Stanford University, Palo Alto, CA, USA. ${ }^{4}$ Sanofi, Chilly-Mazarin, France. ${ }^{5}$ Sanofi Genzyme, Bridgewater, NJ, USA. ${ }^{6}$ Regeneron Pharmaceuticals Inc., Tarrytown, NY, USA.

Received: 8 August 2019 Accepted: 27 March 2020

Published online: 07 April 2020

\section{References}

1. Karmakar S, Kay J, Gravallese EM. Bone damage in rheumatoid arthritis: mechanistic insights and approaches to prevention. Rheum Dis Clin N Am. 2010;36:385-404.

2. Mclnnes IB, Schett G. Cytokines in the pathogenesis of rheumatoid arthritis. Nat Rev Immunol. 2007;7:429-42.

3. Schett G, Gravallese E. Bone erosion in rheumatoid arthritis: mechanisms, diagnosis and treatment. Nat Rev Rheumatol. 2012;8:656-64.

4. Choy E. Understanding the dynamics: pathways involved in the pathogenesis of rheumatoid arthritis. Rheumatology (Oxford). 2012;51(Suppl 5):v3-11.

5. Smolen JS, Aletaha D. The assessment of disease activity in rheumatoid arthritis. Clin Exp Rheumatol. 2010;28:S18-27.

6. Schett $G$. Physiological effects of modulating the interleukin- 6 axis. Rheumatology (Oxford). 2018;57:ii43-50.

7. Calabrese LH, Rose-John S. IL-6 biology: implications for clinical targeting in rheumatic disease. Nat Rev Rheumatol. 2014;10:720-7.

8. Hunter CA, Jones SA. IL-6 as a keystone cytokine in health and disease. Nat Immunol. 2015;16:448-57.

9. Choy EHS, Calabrese LH. Neuroendocrine and neurophysiological effects of interleukin 6 in rheumatoid arthritis. Rheumatology (Oxford). 2018;57: 1885-95.

10. Solomon DH, Goodson NJ, Katz JN, Weinblatt ME, Avorn J, Setoguchi S, et al. Patterns of cardiovascular risk in rheumatoid arthritis. Ann Rheum Dis. 2006;65:1608-12. 
11. Choy E, Ganeshalingam K, Semb AG, Szekanecz Z, Nurmohamed M. Cardiovascular risk in rheumatoid arthritis: recent advances in the understanding of the pivotal role of inflammation, risk predictors and the impact of treatment. Rheumatology (Oxford). 2014;53:2143-54.

12. Gabay C, McInnes IB, Kavanaugh A, Tuckwell K, Klearman M, Pulley J, et al. Comparison of lipid and lipid-associated cardiovascular risk marker changes after treatment with tocilizumab or adalimumab in patients with rheumatoid arthritis. Ann Rheum Dis. 2016;75:1806-12.

13. Genovese MC, Fleischmann R, Kivitz AJ, Rell-Bakalarska M, Martincova R, Fiore $S$, et al. Sarilumab plus methotrexate in patients with active rheumatoid arthritis and inadequate response to methotrexate: results of a phase III study. Arthritis Rheumatol. 2015;67:1424-37.

14. Fleischmann R, van Adelsberg J, Lin Y, Castelar-Pinheiro GD, Brzezicki J, Hrycaj P, et al. Sarilumab and nonbiologic disease-modifying antirheumatic drugs in patients with active rheumatoid arthritis and inadequate response or intolerance to tumor necrosis factor inhibitors. Arthritis Rheumatol. 2017;69:277-90.

15. Burmester GR, Lin Y, Patel R, van Adelsberg J, Mangan EK, Graham NM, et al. Efficacy and safety of sarilumab monotherapy versus adalimumab monotherapy for the treatment of patients with active rheumatoid arthritis (MONARCH): a randomised, double-blind, parallel-group phase III trial. Ann Rheum Dis. 2017;76:840-7.

16. AbbVie Inc. US prescribing information: HUMIRA (adalimumab) injection, solution for subcutaneous use. https://www.accessdata.fda.gov/drugsatfda_ docs/label/2018/125057s410lbl.pdf. Accessed 17 July 2019.

17. Strand V, Gossec L, Proudfoot CWJ, Chen Cl, Reaney M, Guillonneau S, et al. Patient-reported outcomes from a randomized phase III trial of sarilumab monotherapy versus adalimumab monotherapy in patients with rheumatoid arthritis. Arthritis Res Ther. 2018;20:129.

18. Gabay C, Msihid J, Zilberstein M, Paccard C, Lin Y, Graham NMH, et al. Identification of sarilumab pharmacodynamic and predictive markers in patients with inadequate response to TNF inhibition: a biomarker substudy of the phase 3 TARGET study. RMD Open. 2018;4:e000607.

19. Boyapati A, Msihid J, Fiore S, van Adelsberg J, Graham NM, Hamilton JD. Sarilumab plus methotrexate suppresses circulating biomarkers of bone resorption and synovial damage in patients with rheumatoid arthritis and inadequate response to methotrexate: a biomarker study of MOBILITY. Arthritis Res Ther. 2016;18:225.

20. Burmester GR, Hagino O, Dong Q, Stanislav M, Gomez-Centeno A, Selmi C, et al. Unique changes in hemoglobin with sarilumab versus adalimumab are independent of better disease control in patients with rheumatoid arthritis (RA). Arthritis Rheumatol. 2018;70(Suppl. 10):Abstract 1528.

21. Terpos E, Fragiadaki K, Konsta M, Bratengeier C, Papatheodorou A, Sfikakis PP. Early effects of IL-6 receptor inhibition on bone homeostasis: a pilot study in women with rheumatoid arthritis. Clin Exp Rheumatol. 2011;29:921-5.

22. Fassio A, Adami G, Gatti D, Orsolini G, Giollo A, Idolazzi L, et al. Inhibition of tumor necrosis factor-alpha (TNF-alpha) in patients with early rheumatoid arthritis results in acute changes of bone modulators. Int Immunopharmacol. 2019;67:487-9.

23. Song S-NJ, Iwahashi M, Tomosugi N, Uno K, Yamana J, Yamana S, et al. Comparative evaluation of the effects of treatment with tocilizumab and TNF-a inhibitors on serum hepcidin, anemia response and disease activity in rheumatoid arthritis patients. Arthritis Res Ther. 2013;15:R141-R.

24. Gavrilă Bl, Ciofu C, Stoica V. Biomarkers in rheumatoid arthritis, what is new? J Med Life. 2016;9:144-8.

25. Sechidis K, Papangelou K, Metcalfe PD, Svensson D, Weatherall J, Brown G. Distinguishing prognostic and predictive biomarkers: an information theoretic approach. Bioinformatics. 2018;34:3365-76.

26. Kringelbach TM, Glintborg B, Hogdall EV, Johansen JS, Hetland ML. Identification of new biomarkers to promote personalised treatment of patients with inflammatory rheumatic disease: protocol for an open cohort study. BMJ Open. 2018:8:e019325.

27. Dennis G Jr, Holweg CT, Kummerfeld SK, Choy DF, Setiadi AF, Hackney JA, et al. Synovial phenotypes in rheumatoid arthritis correlate with response to biologic therapeutics. Arthritis Res Ther. 2014;16:R90.

28. Curtis JR, Wright GC, Strand V, Davis CS, Hitraya E, Sasso EH. Reanalysis of the multi-biomarker disease activity score for assessing disease activity in the abatacept versus adalimumab comparison in biologic-naive rheumatoid arthritis subjects with background methotrexate study: comment on the article by Fleischmann et al. Arthritis Rheumatol. 2017;69:863-5.

\section{Publisher's Note}

Springer Nature remains neutral with regard to jurisdictional claims in published maps and institutional affiliations.

\section{Ready to submit your research? Choose BMC and benefit from:}

- fast, convenient online submission

- thorough peer review by experienced researchers in your field

- rapid publication on acceptance

- support for research data, including large and complex data types

- gold Open Access which fosters wider collaboration and increased citations

- maximum visibility for your research: over $100 \mathrm{M}$ website views per year

At $\mathrm{BMC}$, research is always in progress.

Learn more biomedcentral.com/submissions 